\title{
A Study on Conjugate Quadrature Filters
}

\author{
Jin-Song Leng, Ting-Zhu Huang, Yan-Fei Jing, and Wei Jiang \\ School of Mathematical Sciences, University of Electronic of Science and Technology of China, \\ Chengdu, Sichuan 610054, China
}

Correspondence should be addressed to Jin-Song Leng, 1-js2004@tom.com

Received 18 June 2010; Revised 26 October 2010; Accepted 5 January 2011

Academic Editor: Antonio Napolitano

Copyright () 2011 Jin-Song Leng et al. This is an open access article distributed under the Creative Commons Attribution License, which permits unrestricted use, distribution, and reproduction in any medium, provided the original work is properly cited.

It is very important for generating an orthonormal multiwavelet system to construct a conjugate quadrature filter (CQF). In this paper, a general method of constructing a length $-J+1$ CQF with multiplicity $r$ and scale $a$ from a length- $J$ CQF is obtained. As a special case, we study generally the construction of a length- $J+1$ CQF with multiplicity 2 and scale 2 which can generate a compactly supported symmetric-antisymmetric orthonormal multiwavelet system from a length-J CQF.

\section{Introduction and Preliminaries}

Wavelet analysis has been proven to be a very powerful tool in harmonic analysis, neural networks, numerical analysis, and signal processing, especially in the area of image compression [1]. Symmetry is a crucial property in signal processing. It is well known that the scalar orthonormal wavelet bases with compact support have no symmetry. Multiwavelets initiated by Goodman et al. [2] overcome the drawback. In practice, orthonormal multiwavelets are of interest because they can be real, compactly supported, continuous, and symmetric. The advantages of multiwavelets and their promising features in applications have attracted a great deal of interest and effort to extensively study them. For example, the advances about multiwavelets can be seen in [3-11]. Yang [9] provided a method of deriving a length$J+1$ conjugate quadrature filter with multiplicity $r$ from a length $J$ conjugate quadrature filter. In this paper, we extend the results of [9] and get a general method of constructing a length $-J+1$ conjugate quadrature filter with multiplicity $r$ and scale $a$ from a length- $J$ conjugate quadrature filter. The corresponding results in [9] are special cases of our results.

Function vectors $\Psi_{l}(x)=\left(\psi_{l, 1}(x), \psi_{l, 2}(x), \ldots, \psi_{l, r}(x)\right)^{T}, l=$ $1,2, \ldots, a-1$ are called orthonormal multiwavelets with scale $a$ associated with an orthonormal multiscaling function vector $\Phi(x)=\left(\phi_{1}(x), \phi_{2}(x), \ldots, \phi_{r}(x)\right)^{T}$ if they generate a multiresolution analysis (MRA) $\left\{V_{j}\right\}_{j \in Z}$ of $L^{2}(R)$ and satisfy the following orthonormal conditions:

$$
\begin{gathered}
\langle\Phi(\cdot-k), \Phi(\cdot-l)\rangle=\delta_{k, l} I_{r}, \\
\left\langle\Psi_{k}(\cdot-m), \Psi_{l}(\cdot-n)\right\rangle=\delta_{k, l} \delta_{m, n} I_{r},
\end{gathered}
$$

and the following refinement equations:

$$
\begin{gathered}
\Phi(x)=\frac{1}{a} \sum_{k \in Z} P_{k} \Phi(a x-k), \\
\Psi_{l}(x)=\frac{1}{a} \sum_{k \in Z} Q_{l, k} \Phi(a x-k), \quad l=1,2, \ldots, a-1,
\end{gathered}
$$

where $\left\{P_{k}\right\}_{k \in Z}$ and $\left\{Q_{l, k}\right\}_{k \in Z}, l=1,2, \ldots, a-1$ are $r \times r$ matrix sequences. The sequence $\left\{P_{k}\right\}_{k \in Z}$ is called low-pass filter, and the sequences $\left\{Q_{l, k}\right\}_{k \in Z}, l=1,2, \ldots, a-1$ are called high-pass filters. $\left\{\Phi(x), \Psi_{1}(x), \Psi_{2}(x), \ldots, \Psi_{a-1}(x)\right\}$ is an orthonormal multiwavelet system generated by these filters. The Fourier transforms of these filters, that is, $P(\omega)=$ $(1 / a) \sum_{k \in Z} P_{k} e^{-i k \omega}$ are $Q_{l}(\omega)=(1 / a) \sum_{k \in Z} Q_{l, k} e^{-i k \omega}, l=$ $1,2, \ldots, a-1$, and $i=\sqrt{-1}$ are called refinement mask and multiwavelet masks, respectively. The orthonormal 
conditions (1) imply the following conditions called the perfect reconstruction $(\mathrm{PR})$ conditions $[2,6]$ :

$$
\begin{aligned}
& \sum_{k=0}^{a-1} P\left(\frac{\omega+2 k \pi}{a}\right) P^{*}\left(\frac{\omega+2 k \pi}{a}\right)=I_{r}, \\
& \sum_{k=0}^{a-1} P\left(\frac{\omega+2 k \pi}{a}\right) Q_{l}^{*}\left(\frac{\omega+2 k \pi}{a}\right)=0_{r}, \\
& l=1,2, \ldots, a-1, \\
& \sum_{k=0}^{a-1} Q_{l}\left(\frac{\omega+2 k \pi}{a}\right) Q_{m}^{*}\left(\frac{\omega+2 k \pi}{a}\right)=\delta_{l, m} I_{r}, \\
& l, m=1,2, \ldots, a-1 .
\end{aligned}
$$

If the sequence $\left\{P_{k}\right\}_{k \in Z}$ satisfies (3), it is called a matrix conjugate quadrature filter (CQF). In this paper, we suppose $P_{k}=0_{r}$ for all $k<0$ or $k>J$, where $J \in Z_{+}$. For the matrix CQF $\left\{P_{k}\right\}_{k=0}^{J}$ with multiplicity $r=2$ and scale $a=2$, the following conditions:

$$
P_{0}, P_{J} \text { are nonzero matrices }
$$

$$
P_{k}=A P_{J-k} A, \quad k=0,1, \ldots, J, \quad \text { where } A=\left[\begin{array}{cc}
1 & 0 \\
0 & -1
\end{array}\right],
$$

$$
P(0)=\left[\begin{array}{ll}
1 & 0 \\
0 & \alpha
\end{array}\right], \quad \text { for some number }|\alpha|<1
$$

are called SA conditions [8]. The condition (7) implies that the corresponding multiscaling function vector forms a symmetric-antisymmetric pair as shown in the following [5]:

$$
\begin{aligned}
P_{k} & =A P_{J-k} A, \quad k=0,1, \ldots, J \\
& \Leftrightarrow P(\omega)=A P(-\omega) A e^{-i J \omega} \\
& \Leftrightarrow \phi_{i}(x)=(-1)^{i-1} \phi_{i}(J-x), \quad i=1,2 .
\end{aligned}
$$

The condition (8) is a necessary condition [7] for a lowpass filter satisfying (6) and (7) to generate a multiresolution analysis.

The paper is organized as follows. In Section 2, we provide a general method of constructing a length- $J+1$ CQF with complicity $r$ and scale $a$ from a length-J CQF. In Section 3, as the application of Section 2, we study generally the construction of a length- $J+1$ CQF with multiplicity 2 and scale 2 which can generate a compactly supported symmetric-antisymmetric orthonormal multiwavelet system from a length- $J$ CQF. In Section 4, we give two numerical examples.

\section{A Study on Matrix Conjugate Quadrature Filters with Arbitrary Multiplicity and Arbitrary Scale}

Lemma 1. Let $M(\omega)$ be a $r \times r$ matrix whose entries are linear polynomials of $e^{-i \omega}$ with real coefficients, then $M(\omega)$ is a unitary matrix if and only if

$$
M(\omega)=M(0)\left(I_{r}-H+H e^{-i \omega}\right),
$$

where the $r \times r$ matrix $M(0)$ satisfies

$$
M(0) M^{T}(0)=I_{r}
$$

and the $r \times r$ matrix $H$ satisfies

$$
H^{T}=H, \quad H^{2}=H .
$$

Proof. It is obvious that the right side of (10) is a unitary matrix. We only prove the forward direction. Assume that $M(\omega)$ is a unitary matrix, then $M(0)$ satisfies (11), and then we have

$$
M(\omega)=M(0)\left(F+H e^{-i \omega}\right)
$$

where $F, H$ are $r \times r$ matrices whose entries are real numbers. Let $\omega=0$, then we have

$$
F=I_{r}-H
$$

Then

$$
\begin{aligned}
M^{*}(\omega) M(\omega) & \\
= & \left(I_{r}-H^{T}+H^{T} e^{i \omega}\right) M^{T}(0) M(0)\left(I_{r}-H+H e^{-i \omega}\right) \\
= & I_{r}-H-H^{T}+2 H^{T} H+\left(H-H^{T} H\right) e^{-i \omega} \\
& +\left(H^{T}-H^{T} H\right) e^{i \omega}=I_{r},
\end{aligned}
$$

which implies

$$
H=H^{T} H=H^{T} .
$$

Hence $H$ satisfies (12).

Theorem 1. Suppose that the $r \times r$ matrix sequence $\left\{P_{O, k}\right\}_{k=0}^{J-1}$ is a length-J CQF, $M$ is an $r \times r$ matrix satisfying (11), and $H$ is an $r \times r$ matrix satisfying (12). Let

$$
P_{N, k}= \begin{cases}P_{O, 0} M\left(I_{r}-H\right), & k=0, \\ P_{O, k-1} M H+P_{O, k} M\left(I_{r}-H\right), & 0<k<J, \\ P_{O, J-1} M H, & k=J,\end{cases}
$$


then the $r \times r$ matrix sequence $\left\{P_{N, k}\right\}_{k=0}^{J}$ is a length-J $+1 C Q F$.

Remark 1. We adopt the subscript " $O$ " in $\left\{P_{O, k}\right\}_{k=0}^{J-1}$ to indicate that it is an old CQF, and adopt the subscript " $N$ " in $\left\{P_{N, k}\right\}_{k=0}^{J}$ to indicate that it is a new CQF.

Proof. Let

$$
M(\omega)=M\left(I_{r}-H+H e^{-i \omega}\right) .
$$

By Lemma 1, $M(\omega)$ is a unitary matrix. Using (17), we have

$$
P_{N}(\omega)=P_{O}(\omega) M(\omega) .
$$

Then

$$
\sum_{k=0}^{a-1} P_{N}\left(\frac{\omega+2 k \pi}{a}\right) P_{N}^{*}\left(\frac{\omega+2 k \pi}{a}\right)=I_{r} .
$$

Therefore, $\left\{P_{N, k}\right\}_{k=0}^{J}$ satisfies (3). Hence $\left\{P_{N, k}\right\}_{k=0}^{J}$ is a length- $J+1 \mathrm{CQF}$.

\section{Some Results on Matrix Conjugate Quadrature Filters with Multiplicity 2 and Scale 2}

Lemma 2 (see [5]). Let $\left\{P_{k}\right\}_{k=0}^{J}$ be a $2 \times 2$ matrix sequence, then the following two statements are equivalent:

(i) $P_{k}=A P_{J-k} A, k=0,1, \ldots, J$, where $A=\left[\begin{array}{cc}1 & 0 \\ 0 & -1\end{array}\right]$.

(ii) $P(\omega)=A P(-\omega) A e^{-i J \omega}$, where $P(\omega)$ is the Fourier transform of $\left\{P_{k}\right\}_{k=0}^{J}$.

Theorem 2. Let $\left\{P_{O, k}\right\}_{k=0}^{J-1}$ be a CQF with multiplicity $r=2$ and scale $a=2$ satisfying $S A$ conditions and let $M, H$ be two $2 \times 2$ matrices satisfying (11) and (12), respectively. Then the new CQF $\left\{P_{N, k}\right\}_{k=0}^{J}$ constructed by (17) satisfies SA conditions if and only if

$$
M=\left[\begin{array}{cc}
1 & 0 \\
0 & \pm 1
\end{array}\right], \quad H=\left[\begin{array}{cc}
\frac{1}{2} & \pm \frac{1}{2} \\
\pm \frac{1}{2} & \frac{1}{2}
\end{array}\right] .
$$

Proof. We first prove the reverse direction. Let

$$
M=\left[\begin{array}{ll}
1 & 0 \\
0 & 1
\end{array}\right], \quad H=\left[\begin{array}{ll}
\frac{1}{2} & \frac{1}{2} \\
\frac{1}{2} & \frac{1}{2}
\end{array}\right],
$$

then

$$
M(\omega)=\left[\begin{array}{cc}
\frac{1}{2}+\frac{1}{2} e^{-i \omega} & -\frac{1}{2}+\frac{1}{2} e^{-i \omega} \\
-\frac{1}{2}+\frac{1}{2} e^{-i \omega} & \frac{1}{2}+\frac{1}{2} e^{-i \omega}
\end{array}\right],
$$

which satisfies

$$
A M(\omega)=M(-\omega) A e^{-i \omega}
$$

Using Lemma 2 as well as (19) and (23), we have

$$
P_{N}(\omega)=P_{O}(\omega) M(\omega)=A P_{N}(-\omega) A e^{-i J \omega} .
$$

Hence $\left\{P_{N, k}\right\}_{k=0}^{J}$ satisfies (7) by Lemma 2. Because $\left\{P_{O, k}\right\}_{k=0}^{J-1}$ satisfies (8), we have

$$
P_{N}(0)=P_{O}(0) M(0)=P_{O}(0)=\left[\begin{array}{ll}
1 & 0 \\
0 & \alpha
\end{array}\right],
$$

for some number $|\alpha|<1$.

Hence $\left\{P_{N, k}\right\}_{k=0}^{J}$ satisfies (8). It is obvious that $\left\{P_{N, k}\right\}_{k=0}^{J}$ satisfies (6). So $\left\{P_{N, k}\right\}_{k=0}^{J}$ satisfies SA conditions. The proof of the other cases of $M, H$ in (21) is similar.Then we prove the forward direction. Suppose that the new CQF $\left\{P_{N, k}\right\}_{k=0}^{J}$ constructed by (17) satisfies SA conditions. Let $M=\left[\begin{array}{ll}a & b \\ c & d\end{array}\right]$, then

$$
P_{N}(0)=P_{O}(0) M=\left[\begin{array}{cc}
a & b \\
\alpha c & \alpha d
\end{array}\right]
$$

which satisfies (8). Noting that $M$ satisfies (11), we can get

$$
a=1, \quad d= \pm 1, \quad b=c=0 .
$$

Hence

$$
M=\left[\begin{array}{cc}
1 & 0 \\
0 & \pm 1
\end{array}\right]
$$

Because $H$ satisfies (12), we have

$$
H=\left[\begin{array}{cc}
a & \pm \sqrt{a-a^{2}} \\
\pm \sqrt{a-a^{2}} & 1-a
\end{array}\right], \quad \text { or } \quad H=I_{r}, \quad a>0 .
$$

Hence

$$
\begin{aligned}
& M(\omega) \\
& =M\left[\begin{array}{cc}
1-a+a e^{-i \omega} & \mp \sqrt{a-a^{2}} \pm \sqrt{a-a^{2}} e^{-i \omega} \\
\sqrt{a-a^{2}} \pm \sqrt{a-a^{2}} e^{-i \omega} & a+(1-a) e^{-i \omega}
\end{array}\right], \\
& \text { or } M(\omega)=M e^{-i \omega} .
\end{aligned}
$$

Because $\left\{P_{N, k}\right\}_{k=0}^{J}$ satisfies (7), by Lemma 2 and (19), we have

$$
P_{N}(\omega)=A P_{N}(-\omega) A e^{-i J \omega}=A P_{O}(-\omega) A M(\omega) e^{-i(J-1) \omega} .
$$

Hence

$$
A M(\omega)=M(-\omega) A e^{-i \omega}
$$




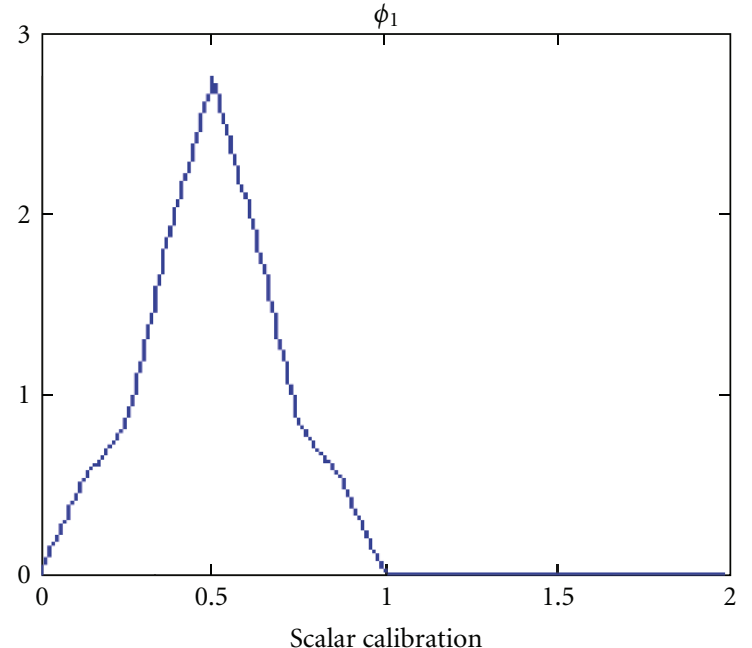

(a)

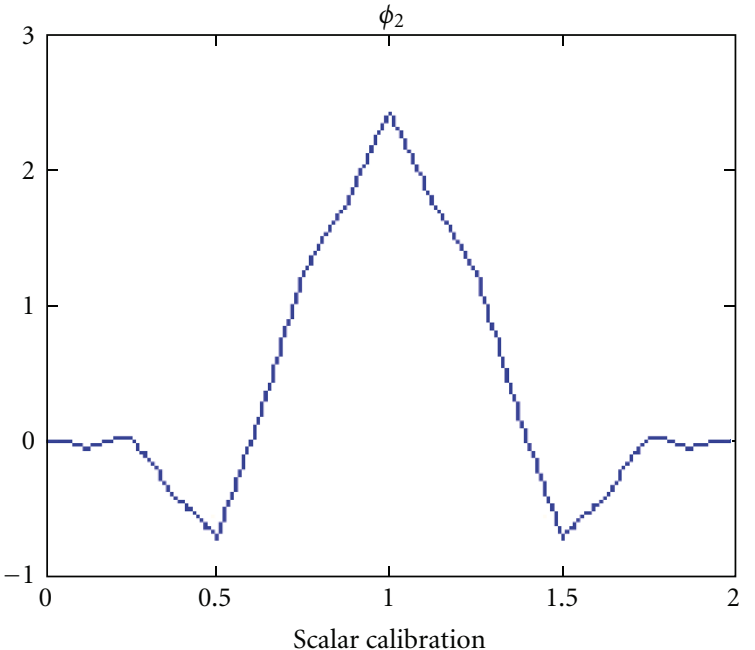

(b)

FIGURE 1: The scaling functions on interval $[0,2]$ generated by the new lengh- 5 low-pass filter.

Using (31) and (33), we have

$$
H=\left[\begin{array}{cc}
\frac{1}{2} & \pm \frac{1}{2} \\
\pm \frac{1}{2} & \frac{1}{2}
\end{array}\right]
$$

Similarly to the above theorem, we can get the following theorem.

Theorem 3. Suppose that $\left\{P_{O, k}\right\}_{k=0}^{J-1}$ and $\left\{Q_{O, l, k}\right\}_{k=0}^{L-1}, \quad l=$ $1,2, \ldots, a-1, \quad L \in Z_{+}$generate a symmetric-antisymmetric orthonormal multiwavelet system with multiplicity $r=2$ and scale $a=2 .\left\{P_{N, k}\right\}_{k=0}^{J}$ and $\left\{Q_{N, l, k}\right\}_{k=0}^{L}, \quad l=1,2, \ldots, a-1$ are given by (17) from $\left\{P_{O, k}\right\}_{k=0}^{J-1}$ and $\left\{Q_{O, l, k}\right\}_{k=0}^{L-1}$, respectively. Then $\left\{P_{N, k}\right\}_{k=0}^{J}$ and $\left\{Q_{N, l, k}\right\}_{k=0}^{L}, \quad l=1,2, \ldots, a-1$ generate a new symmetric-antisymmetric orthonormal multiwavelet system.

\section{Numerical Examples}

Example 1. The following CQF was constructed in [3] by fractal interpolation:

$$
\begin{aligned}
& P_{0}=\frac{1}{20}\left[\begin{array}{cc}
12 & 16 \sqrt{2} \\
-\sqrt{2} & -6
\end{array}\right], \quad P_{1}=\frac{1}{20}\left[\begin{array}{cc}
12 & 0 \\
9 \sqrt{2} & 20
\end{array}\right], \\
& P_{2}=\frac{1}{20}\left[\begin{array}{cc}
0 & 0 \\
9 \sqrt{2} & -6
\end{array}\right], \quad P_{3}=\frac{1}{20}\left[\begin{array}{cc}
0 & 0 \\
-\sqrt{2} & 0
\end{array}\right] .
\end{aligned}
$$

Let

$$
M=\left[\begin{array}{cc}
\frac{1}{2} & -\frac{\sqrt{3}}{2} \\
\frac{\sqrt{3}}{2} & \frac{1}{2}
\end{array}\right], \quad H=\left[\begin{array}{cc}
\frac{1}{4} & -\frac{\sqrt{3}}{4} \\
-\frac{\sqrt{3}}{4} & \frac{3}{4}
\end{array}\right] \text {, }
$$

we get the following new length-5 CQF with (17):

$$
\begin{aligned}
& \bar{P}_{0}=\frac{1}{20}\left[\begin{array}{cc}
8 \sqrt{6} & 8 \sqrt{2} \\
-3 \sqrt{3} & -3
\end{array}\right], \\
& \bar{P}_{1}=\frac{1}{20}\left[\begin{array}{cc}
6 & -6 \sqrt{3} \\
10 \sqrt{3}-\frac{\sqrt{2}}{2} & 10+\frac{\sqrt{6}}{2}
\end{array}\right], \\
& \bar{P}_{2}=\frac{1}{20}\left[\begin{array}{cc}
6 & -6 \sqrt{3} \\
-3 \sqrt{3}+\frac{9 \sqrt{2}}{2} & -3-\frac{9 \sqrt{6}}{2}
\end{array}\right], \\
& \bar{P}_{3}=\frac{1}{20}\left[\begin{array}{cc}
0 & 0 \\
\frac{9 \sqrt{2}}{2} & -\frac{9 \sqrt{6}}{2}
\end{array}\right], \\
& \bar{P}_{4}=\frac{1}{20}\left[\begin{array}{cc}
0 & 0 \\
-\frac{\sqrt{2}}{2} & \frac{\sqrt{6}}{2}
\end{array}\right] .
\end{aligned}
$$

The scaling functions generated by the lengh-5 low-pass filter are shown in Figure 1.

In signal processing, an original signal is decomposed by the low-pass filters and the high-pass filters into different frequency components, and then each component with a resolution matched to its scale is studied. Given a test signal which is the function

$$
f(t)=\sin t+\sin 3 t+\sin 5 t
$$

we decompose the test signal with the old lengh-4 low-pass filter and the new lengh-5 low-pass filter of the example. The results are shown in Figure 2. 


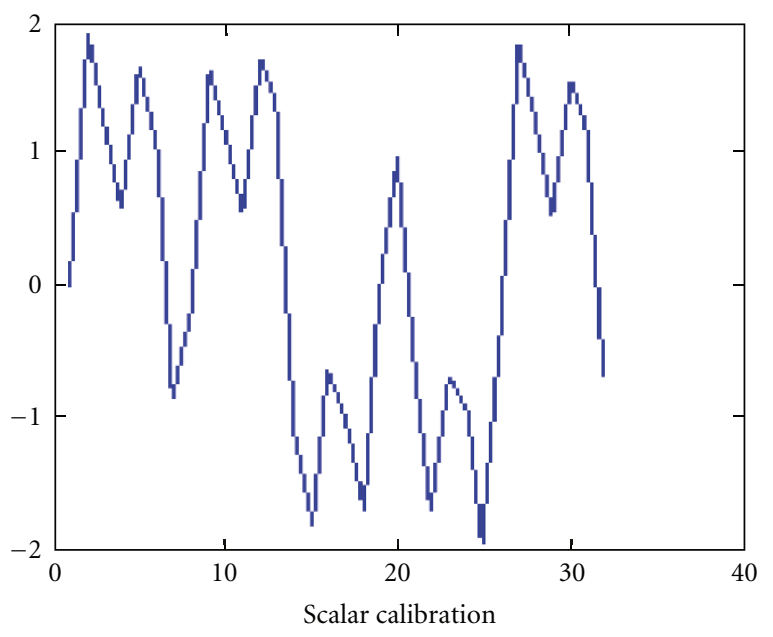

(a) The test signal

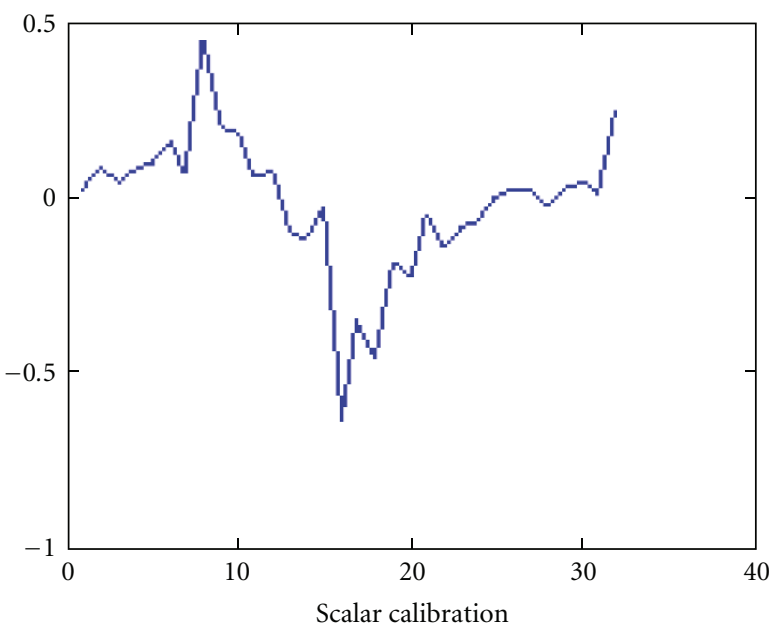

(b) Decomposition with the old lengh-4 low-pass filter

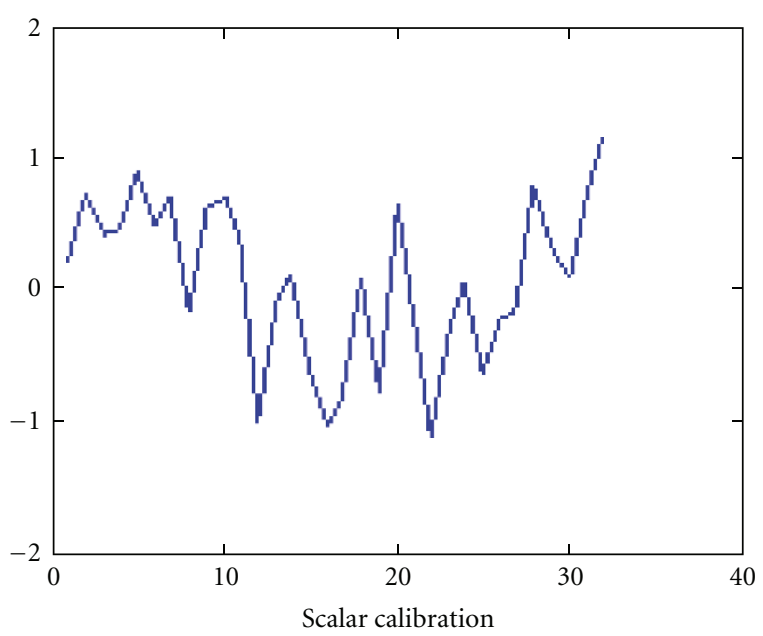

(c) Decomposition with the new lengh-5 low-pass filter

Figure 2: Decompositions of the test signal, prefiltered with Haar on interval $[0,40]$.

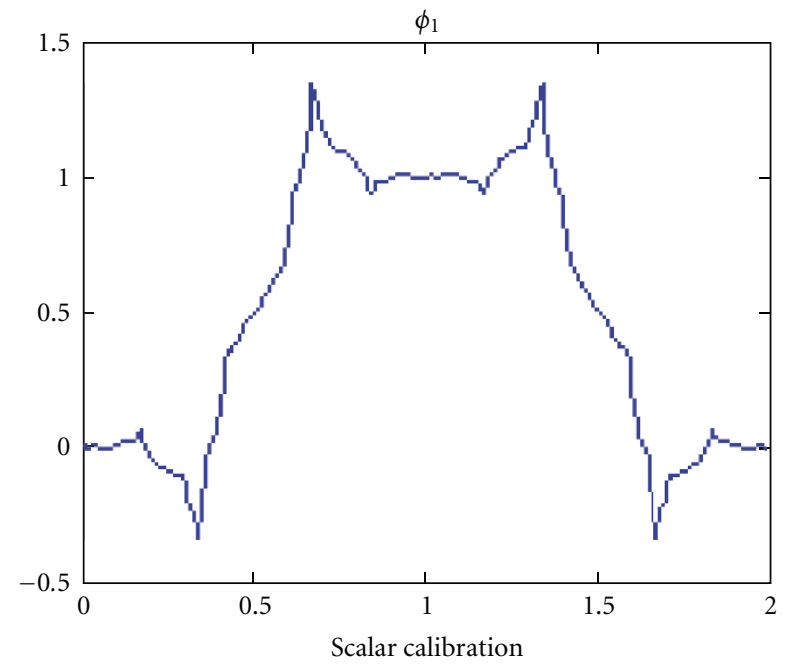

(a)

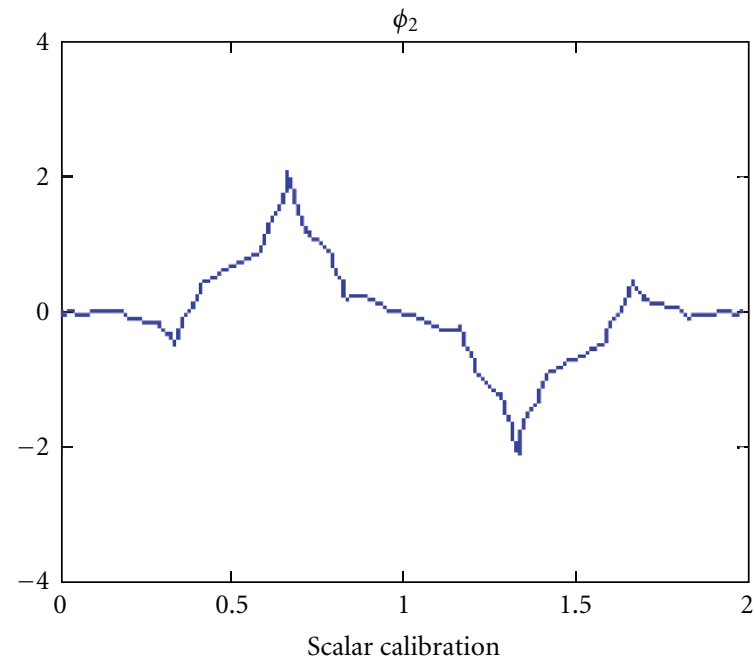

(b)

Figure 3: The scaling functions on interval $[0,2]$ generated by the lengh-3 low-pass filter with $\theta=\pi / 6$. 


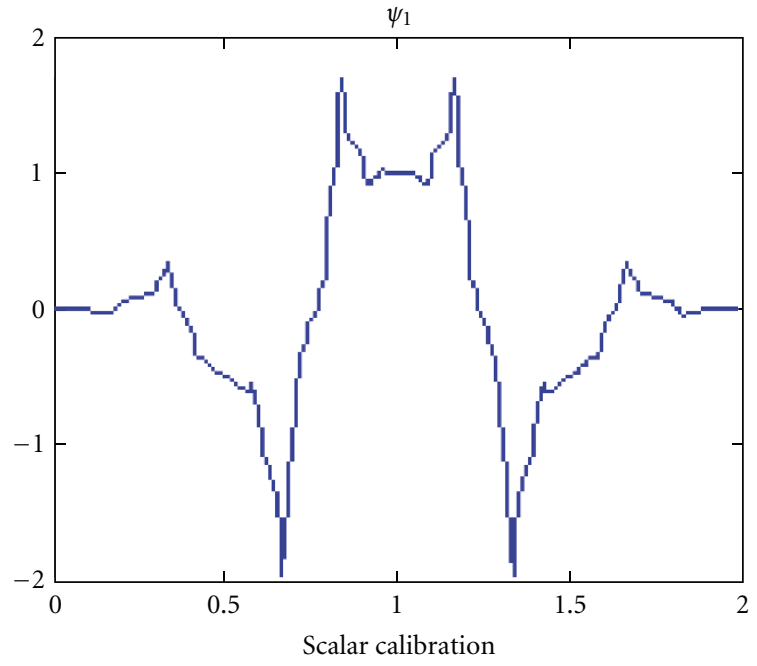

(a)

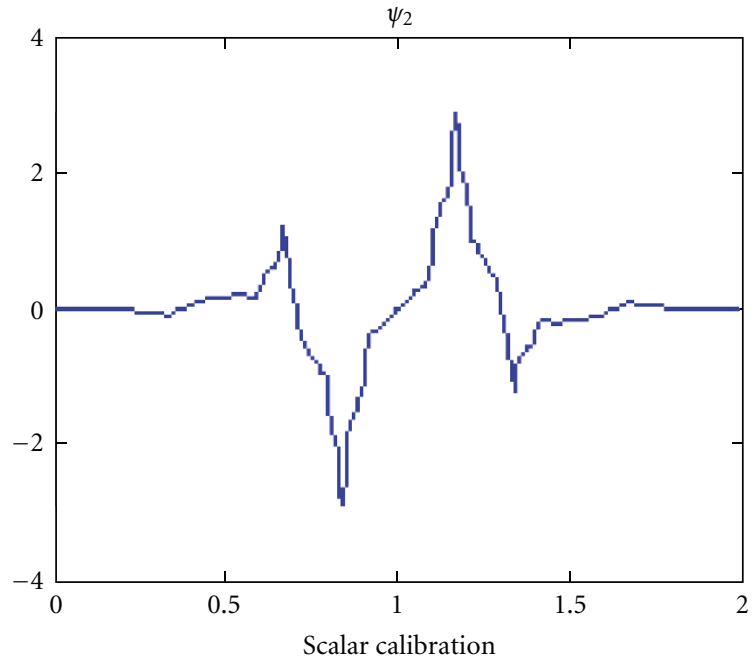

(b)

FIGURE 4: The wavelet functions on interval $[0,2]$ generated by the lengh- 3 high-pass filter with $\theta=\pi / 6$.

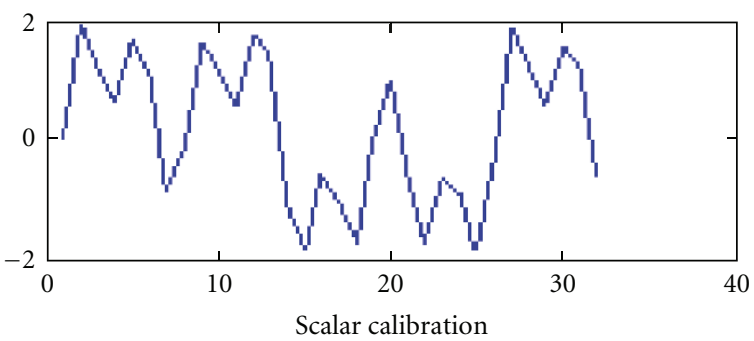

(a) The test signal

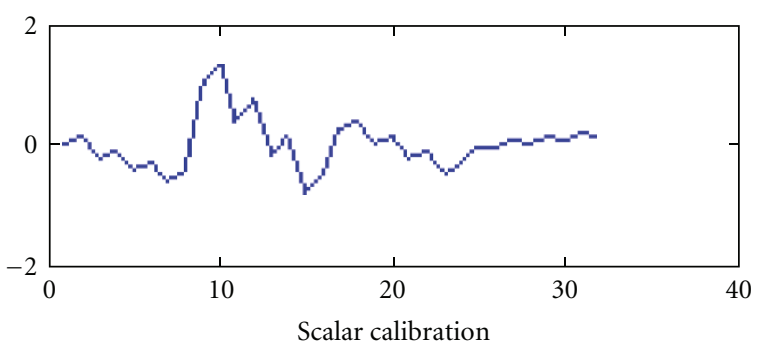

(c) Decomposition with the old lengh-2 high-pass filter

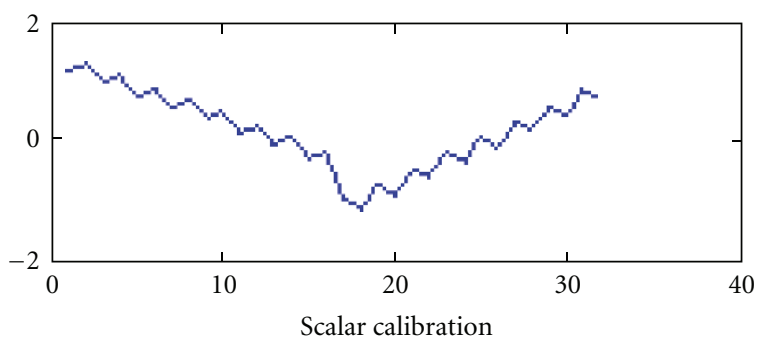

(b) Decomposition with the old lengh-2 low-pass filter

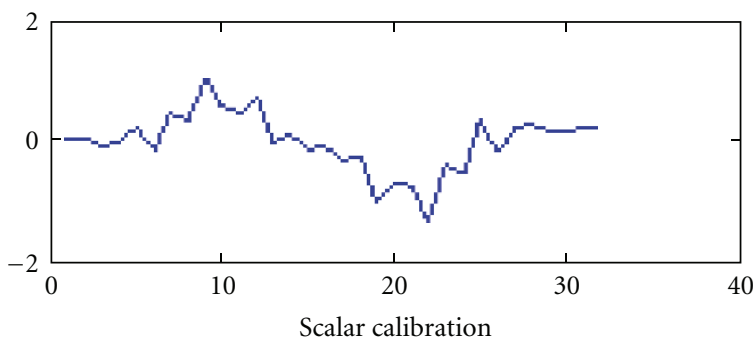

(d) Decomposition with the new lengh-3 low-pass filter

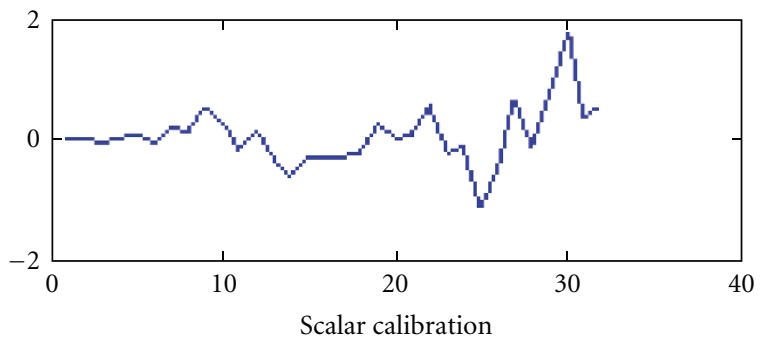

(e) Decomposition with the new lengh-3 high-pass filter

FIgURE 5: Decompositions of the test signal, prefiltered with Haar on interval $[0,40]$. 
Example 2. The following lengh-2 low-pass filter and high-pass filter constructed in [8] generate a symmetricantisymmetric orthonormal multiwavelet system with multiplicity $r=2$ and scale $a=2$ :

$$
\begin{array}{ll}
P_{0}=\left[\begin{array}{cc}
1 & 0 \\
\cos \theta & \sin \theta
\end{array}\right], & P_{1}=\left[\begin{array}{cc}
1 & 0 \\
-\cos \theta & \sin \theta
\end{array}\right], \\
Q_{0}=\left[\begin{array}{cc}
0 & -1 \\
-\sin \theta & \cos \theta
\end{array}\right], & Q_{1}=\left[\begin{array}{cc}
0 & 1 \\
\sin \theta & \cos \theta
\end{array}\right],
\end{array}
$$

where $\theta \in[0,2 \pi) \backslash\{\pi / 2,3 \pi / 2\}$. Let $M=\left[\begin{array}{cc}1 & 0 \\ 0 & -1\end{array}\right], H=$ $\left[\begin{array}{ll}1 / 2 & 1 / 2 \\ 1 / 2 & 1 / 2\end{array}\right]$, then we can get the following lengh-3 low-pass filter and high-pass filter which generate a new symmetricantisymmetric orthonormal multiwavelet system using (17):

$$
\begin{gathered}
\bar{P}_{0}=\left[\begin{array}{cc}
\frac{1}{2} & -\frac{1}{2} \\
\frac{\sqrt{2}}{2} \cos \gamma & -\frac{\sqrt{2}}{2} \cos \gamma
\end{array}\right], \quad \bar{P}_{1}=\left[\begin{array}{cc}
1 & 0 \\
0 & \sqrt{2} \sin \gamma
\end{array}\right], \\
\bar{P}_{2}=\left[\begin{array}{cc}
\frac{1}{2} & \frac{1}{2} \\
-\frac{\sqrt{2}}{2} \cos \gamma & -\frac{\sqrt{2}}{2} \cos \gamma
\end{array}\right], \\
\bar{Q}_{0}=\left[\begin{array}{c}
-\frac{1}{2} \\
\frac{\sqrt{2}}{2} \sin \gamma-\frac{\sqrt{2}}{2} \sin \gamma
\end{array}\right], \quad \bar{Q}_{1}=\left[\begin{array}{cc}
1 & 0 \\
0 & -\sqrt{2} \cos \gamma
\end{array}\right], \\
\bar{Q}_{2}=\left[\begin{array}{cc}
-\frac{1}{2} & -\frac{1}{2} \\
-\frac{\sqrt{2}}{2} \sin \gamma & -\frac{\sqrt{2}}{2} \sin \gamma
\end{array}\right],
\end{gathered}
$$

where $\gamma=\pi / 4-\theta, \theta \in[0,2 \pi) \backslash\{\pi / 2,3 \pi / 2\}$.

The scaling functions and the wavelet functions generated by the lengh-3 low-pass filter and high-pass filter with $\theta=\pi / 6$ are displayed in Figures 3 and 4, respectively.

We compose the test signal of the above example with the old lengh-2 low-pass filter and high-pass filter and the new lengh-3 low-pass filter and high-pass filter with $\theta=\pi / 6$ of this example. The results are shown in Figure 5.

\section{Conclusion}

In this paper, a general method of constructing a length$J+1$ conjugate quadrature filter with multiplicity $r$ and scale $a$ from a length- $J$ CQF is presented. As an application of this result, a method is proposed for constructing a length- $J+1$ CQF with multiplicity 2 and scale 2 which can generate a compactly supported symmetric-antisymmetric orthonormal multiwavelet system from a length-J CQF. The proposed results are more general than the corresponding results of [9]. Finally, two numerical examples are given to verify our results.

\section{Acknowledgment}

The authors wish to thank the anonymous reviewers for their valuable comments and suggestions to improve the presentation of this paper.

\section{References}

[1] A. Said and W. A. Pearlman, "A new, fast, and efficient image codec based on set partitioning in hierarchical trees," IEEE Transactions on Circuits and Systems for Video Technology, vol. 6, no. 3, pp. 243-250, 1996.

[2] T. N. T. Goodman, S. L. Lee, and W. S. Tang, "Wavelet in wandering subspaces," Transactions of the American Mathematical Society, vol. 338, pp. 639-654, 1993.

[3] J. S. Geronimo, D. P. Hardin, and P. R. Massopust, "Fractal functions and wavelet expansions based on several scaling functions," Journal of Approximation Theory, vol. 78, no. 3, pp. 373-401, 1994.

[4] V. Strela, Multiwavelets: theory and applications, Ph.D. thesis, Massachusetts Institute of Technology, 1996.

[5] C. K. Chui and J. A. Lian, "A study of orthonormal multiwavelets," Applied Numerical Mathematics, vol. 20, no. 3, pp. 273-298, 1996.

[6] Z. Shen, "Refinable function vectors," SIAM Journal on Mathematical Analysis, vol. 29, no. 1, pp. 235-250, 1998.

[7] Q. Jiang and Z. Shen, "On existence and weak stability of matrix refinable functions," Constructive Approximation, vol. 15, no. 3, pp. 337-353, 1999.

[8] L. Shen, H. H. Tan, and J. Y. Tham, "Symmetricantisymmetric orthonormal multiwavelets and related scalar wavelets," Applied and Computational Harmonic Analysis, vol. 8, no. 3, pp. 258-279, 2000.

[9] S. Z. Yang, Constructions and algorithms and applications of wavelets, Ph.D. thesis, Xi'an Jiaotong University, 2001.

[10] J. K. Zhang, T. N. Davidson, Z. Q. Luo, and K. M. Wong, "Design of interpolating biorthogonal multiwavelet systems with compact support," Applied and Computational Harmonic Analysis, vol. 11, no. 3, pp. 420-438, 2001.

[11] D. X. Zhou, "Interpolatory orthogonal multiwavelets and refinable functions," IEEE Transactions on Signal Processing, vol. 50, no. 3, pp. 520-527, 2002. 